\title{
Landmark navigation in gerbils: The role of the posterior parietal cortex
}

\author{
COLIN G. ELLLARD \\ University of Waterloo, Waterloo, Ontario, Canada
}

\begin{abstract}
Gerbils were trained to find targets by using visible intramaze landmarks. Normal gerbils were able to find targets by using unstable landmarks, either with or without an externally defined directional vector. Following lesions of the posterior parietal cortex, the gerbils had no clear deficit in navigating with intramaze landmarks but were significantly impaired in a variant of the task that required that they use only extramaze landmarks. These findings confirm and extend recent evidence regarding rodents' abilities to navigate by using landmarks, and they suggest that the posterior parietal cortex is preferentially involved in navigating to places that are defined by arrays of distal landmarks.
\end{abstract}

In a classic series of experiments, Collett, Cartwright, and Smith (1986) showed that it was possible to train gerbils to find a location in space that was defined by a series of one or more visual landmarks. By means of a number of ingenious manipulations of the relative positions of the landmarks, targets, and starting positions, Collett et al. shed some light on the properties of the spatial representations that gerbils use to perform this task. In brief, they showed that gerbils computed the location of the target relative to each of a number of local landmarks and then arrived at a best-fit solution for target location. Although Collett et al. and others who have looked explicitly at landmark navigation in rodents (Biegler \& Morris 1993, 1996a, 1996b) have garnered strong evidence for the ability of these animals to use visual landmarks to aid navigation, there is no evidence that an array of landmarks can be used to find a target when not supplemented by other sources of navigational information, such as compass directions derived from geometry (Cheng, 1986), distal visual information (Suzuki, Augerinos, \& Black, 1980), and information from self-motion (Mittelstaedt \& Mittelstaedt, 1980).

Recent experiments by Biegler and Morris (1993, 1996a, 1996b) have addressed these shortcomings. They argued that an object in the environment is only useful as a landmark if its location is stable. In some conditions in which landmarks move about from trial to trial (and, thereby, come to conflict with other sources of navigational information), they exert relatively poor control over target searching, even if they predict the location of a target more reliably than any other single source of information. In their most recent report, Biegler and Morris (1996a)

This research was supported by a grant from the Natural Sciences and Engineering Research Council of Canada. The author thanks Ina Kulczynska, Cristina Gojmerac, John Robinson, Marla Bigel, Kathy Blom, and Nancy Gibson for their contributions to these experiments. Correspondence concerning this article should be addressed to $\mathrm{C}$. G. Ellard, Department of Psychology, University of Waterloo, Waterloo, ON, N2L 3GI Canada (e-mail: cellard@watarts.uwaterloo.ca). tested the ability of rats to find a target relative to either one or two distinct landmarks while most other sources of navigational information were eliminated or randomized. Rats were disoriented by spinning prior to testing, and the test field consisted of a square box with a single white wall, whose location in the room was varied randomly from trial to trial. In these conditions, rats were not able to derive a compass direction from the very salient white wall and so did not perform well in a single landmark test, but they could use an array of two spaced landmarks to find the target, presumably by using the stable relationship between the two landmarks to derive target direction. In another condition that did not involve vestibular disorientation, rats were able to navigate to the target location by using either a single landmark or an array of two landmarks.

Although the Biegler and Morris (1993, 1996a, 1996b) experiments came close to eliminating all other sources of navigational information, they appear not to have eliminated one potentially useful source of directional information. Although they randomized the relationship between the single white wall of the testing field and the location of the two-landmark array, they appear to have varied only the distance of the landmark array from the walls of the test field, and not its orientation. Given other evidence for the power of the geometry of the test apparatus to exert an influence on rodent navigation (Cheng, 1986; Margules \& Gallistel, 1988), it is possible that the rats in this experiment were able to use the angle between the array of landmarks and the closest wall of the chamber as a source of information about target location. By rotating the landmark array randomly from trial to trial and, thereby, eliminating this source of information, one goal of the present study was to determine whether gerbils could find a target when only the location and orientation of the landmarks cued the location of the target.

Along with the continuing strong interest in the functional basis of navigation in rodents, great attention has been paid to the neural underpinnings of these behaviors. Much of this attention has been focused on the role of 
the hippocampus, in part because of the discovery of place cells (O'Keefe, 1976) and because of a host of other anatomical, physiological, and behavioral findings that suggest that the hippocampus may play some special role in certain aspects of navigation (e.g., see reviews by McNaughton et al., 1996; O'Keefe \& Nadel, 1978). Although they have not received quite the same degree of attention in this context, there is a fairly strong consensus that cortical regions contribute to navigational performance in rodents. In particular, lesion evidence suggests that the parietal cortex contributes to performance in traditional spatial tasks that involve a navigational component, such as the Morris maze (Kolb, Buhrmann, McDonald, \& Sutherland, 1994; Kolb \& Walkey, 1987), the holeboard (Kesner, Farnworth, \& DiMattia, 1989), and the radial maze (Kesner, DiMattia, \& Crutcher, 1987; Kolb \& Walkey, 1987). In addition, rats with parietal lesions show deficits in nonassociative spatial tasks, such as reaction-to-novelty tests (Save, Poucet, Foreman, \& Buhot, 1992). Although the effects of parietal lesions on a very wide variety of spatial tasks have been reported (see reviews by Kolb, 1990, and Thinus-Blanc, 1996), there has not been a full account of the effects of such lesions on variants of the landmark-learning tasks designed by Collett et al. (1986). A second aim of the present paper was to make the first such report.

The first two experiments in the present paper report the outcome of behavioral experiments designed to test the abilities of gerbils to use unstable local landmarks to find a target. In the first experiment, a landmark must be used in conjunction with a compass direction, presumably derived from uncontrolled intra- and extramaze cues. In the second experiment, all intra- and extramaze cues were made irrelevant by requiring gerbils to learn the location of a target relative to two local landmarks whose relationship to one another was fixed but whose relationship to all other sources of navigational information was completely unstable. We found that gerbils were able to complete both types of navigational task with high accuracy.

Experiments 3-5 were designed to explore the effects of parietal lesions on performance in the landmark tasks described above. In general, we found that parietal lesions did not produce deficits in landmark learning, despite the fact that such lesions produced large deficits in an open field navigation task that did not require local landmark information.

\section{EXPERIMEN'T 1}

In the first experiment, gerbils were trained to distinguish between a positive and a negative landmark and to find a location that was a constant distance and direction from the positive landmark. A similar task has been employed by Biegler and Morris (1993), but our task differed from theirs in that there was no stable relationship between the two landmarks or between the distances of ei- ther landmark to the walls of the apparatus or the distal sensory environment.

\section{Method}

Subjects. Eight male Mongolian gerbils (Meriones unguiculatus) were the subjects in this experiment. The gerbils were bred in the laboratory from stock provided by High Oak Breeding Ranch (Guelph, Ontario) and were housed in pairs until the beginning of the experiment in clear polyethylene cages on a 12:12-h light:dark cycle. All behavioral testing was carried out during the light phase. During training and testing, the gerbils were food restricted and were maintained at approximately $90 \%$ of their free-feeding weight.

Apparatus. The testing field was a wooden box $(78 \times 78 \mathrm{~cm})$ with walls $31 \mathrm{~cm}$ high. The floor of the box was covered to a depth of about $2 \mathrm{~cm}$ with woodchips, and it was placed underneath a mirror angled at $45^{\circ}$. All the trials were videotaped by aiming a camera into the mirror. The landmarks consisted of two cylinders. One cylinder (diameter, $4 \mathrm{~cm}$; height, $30 \mathrm{~cm}$ ) was made of stainless steel and had a highly polished silver appearance. The other cylinder (diameter, $5 \mathrm{~cm}$; height, $29 \mathrm{~cm}$ ) was made of plastic and was flat gray.

Procedure. At the beginning of training, each gerbil was placed into the box for a period of about $30 \mathrm{~min}$ for 3 consecutive days, in order to habituate it to the apparatus. On the 4th day, the landmarks and some very small morsels of lab chow were placed onto the floor of the apparatus, and each gerbil was allowed to explore the floor and eat the food. Beginning on the 5th day, the gerbils received 10 training trials/day. On each training trial, the two landmarks were placed at two random locations within the box, the only constraint being that neither of the two landmarks was closer than $10 \mathrm{~cm}$ to any wall. Locations were determined by generating a series of random $x$ - and $y$-coordinates, using a computer program, and placing each landmark at the location in the box indicated by the random numbers. For each gerbil, one of the landmarks was designated $\mathrm{L}+$, and the other $\mathrm{L}-$. On each trial, a small morsel of lab chow was placed at a distance $10 \mathrm{~cm}$ south of the landmark (not exactly due south, but approximately south, and always in the same direction, relative to the walls of the testing room). The experimenter stood in the same location during each trial (which we ensured by marking a small $x$ on the floor). This was considered to be quite important, since the experimenter's head was in clear view of the animal during all the trials and would likely have served as a prominent extramaze landmark. The gerbil was placed in the box at a random location (in one of the corners or in the middle of one of the four sides of the box) and was left in the box for a period of $2 \mathrm{~min}$ or until it found and began to eat the food. On early training days, woodchips in the vicinity of the target were removed in order to facilitate learning of the relationship between the landmark and the target. On later trials, the food was buried just underneath the woodchips. Each gerbil continued to receive daily training until it could reliably and consistently find the food within $10 \mathrm{sec}$ of introduction to the box on $75 \%$ of the training trials. Training to criterion took about $25-30$ sessions. Following training, gerbils received 3 sessions of formal testing. In each test session, the gerbils received 8 trials. Six of the trials were the same as the training trials, and 2 randomly selected trials were probe trials. On probe trials, no food was placed at the target location. The gerbils were placed into the test box for a period of $30 \mathrm{sec}$, and search patterns were videotaped for later analysis. In this and all subsequent experiments, only the results from probe trials (with no food) were analyzed, in order to eliminate the possibility of olfactory cues that might guide target searches. To further this aim, the woodchips in the apparatus were frequently churned around by hand, in order to avoid buildup of odor trails.

Analysis. Search patterns were quantified by placing an acetate overlay on the screen of a video monitor and advancing the videotape in 1-sec increments. At each increment, the position of the 


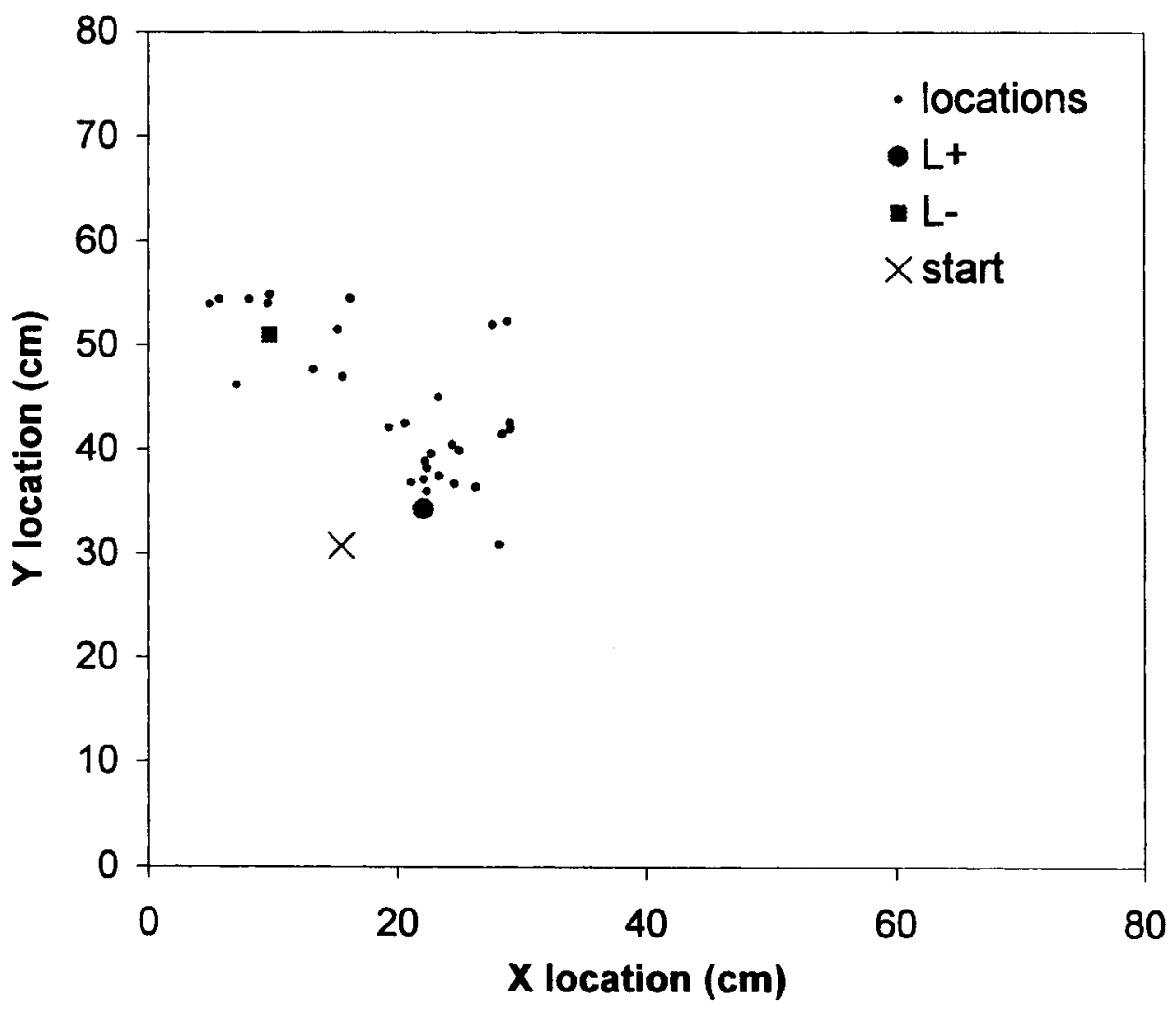

Figure 1. A typical search pattern of 1 gerbil on one 30-sec probe trial in Experiment 1. The locations of the positive and negative landmarks are indicated by the textured circle and square, respectively. The location of the starting position of the gerbil is indicated by the $X$. Each small point represents the sampled location of the gerbil in a 1-sec interval. South is toward the top of the figure (this convention was adopted because of the mirror reversal of images of the apparatus that resulted from our setup). The figure shows a clustering of points on the south side of the positive landmark.

gerbil's snout was marked on the overlay with a marker. Following this, acetate sheets were digitized, using a flatbed scanner, and $x y$ coordinates were extracted, using NIH Image. I

\section{Results and Discussion}

Visual inspection of the results of an analysis of the time-sampled locations of animals over the $30 \mathrm{sec}$ of the probe trials made it clear that the gerbils were not only searching near the correct landmark but were also concentrating their searches on the correct side of the landmark. Figure 1 shows an example of a typical search pattern by 1 gerbil on one of the six trials. To verify statistically that the gerbils were searching at the correct location, we measured the distance of each time-sampled location from each of three points: $10 \mathrm{~cm}$ south of the correct target (the target; this was the rewarded location during training), $10 \mathrm{~cm}$ north of the correct target (the antitarget), and $10 \mathrm{~cm}$ south of the incorrect target (the wrong target). We counted the number of time samples in which the gerbils were less than $5 \mathrm{~cm}$ from each of these three locations, and these totals, converted to time in seconds, were used as the dependent measure in a two-way repeated measures analy- sis of variance (ANOVA). As is shown in Figure 2, the gerbils spent more than half of the 30 -sec trial interval searching within $5 \mathrm{~cm}$ of the correct target location and significantly less time searching at either the antitarget or the wrong target location. The results of the ANOVA showed a significant effect of search location $[F(2,12)=$ $20.457, p<.001]$, but neither the trial nor the trial $\times$ location interaction was significant. In order to compute the trial effects, values from the two probe trials conducted on each day were averaged. A post hoc analysis (Tukey) revealed that all of the observed differences between means were significant $(p<.01)$. Hence, not only did the animals successfully discriminate between the target and the antitarget positions, but they were more likely to search at the antitarget position than at the wrong target position.

These findings suggest that gerbils can learn to search at a location that is defined by a radially symmetric landmark and a compass direction, even when the metric relationship between the landmark, the distal room cues, the geometry of the testing field, and the starting location of the animal are all randomized from trial to trial These 


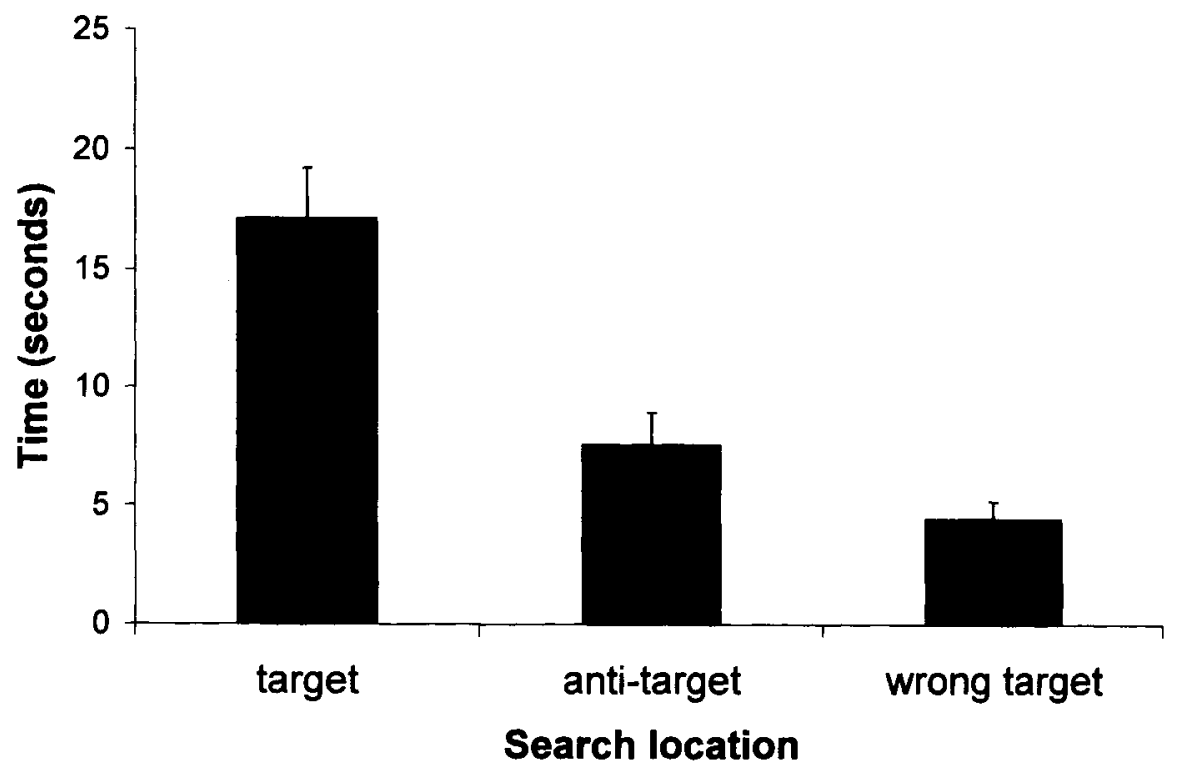

Figure 2. A histogram showing the average time spent within $5 \mathrm{~cm}$ of the target in Experiment 1 , as compared with time spent within $5 \mathrm{~cm}$ of the antitarget location $(5 \mathrm{~cm}$ north of the positive landmark) and of the wrong target location $(5 \mathrm{~cm}$ south of the negative landmark). Each bar shows the average composed of all the probe trials over all the testing days. Error bars represent standard errors of the mean.

findings are counter to earlier arguments of Biegler and Morris (1993) that, unless a stable relationship exists between a landmark and some aspect of the distal environment, animals can learn to use an object only as a beacon to locate an object and not as a true landmark (in the sense that a landmark indicates the location of an object at a distance). In our experiment, evidence that the landmark is not serving as a simple beacon comes from the significant difference between search times at target and antitarget locations. The present evidence is somewhat more in accord with later arguments of Biegler and Morris (1996a, 1996b), who suggested that, under some conditionsparticularly, when all the sources of navigational information (geometry, distal sensory cues, vestibular cues) are internally consistent-rats can navigate by using a single, unstable landmark. Biegler and Morris (1996a, 1996b) have suggested that one contribution to directional information that might have influenced the outcome of their earlier studies was the maintenance of a consistent spatial relationship between the positive and the negative landmarks. Our finding suggests that gerbils are still able to find direction when the spatial relationship between landmarks is inconstant.

\section{EXPERIMENT 2}

In the first experiment, we found strong evidence that gerbils can use a single landmark to find a target even when the relationship between the location of the landmark and all other sources of navigational information is unpredictable. As Biegler and Morris (1996a) argue, it is most likely that this is because, even though landmark locations were completely unpredictable from trial to trial, other sources of navigational information, by remaining consistent, produced a strong compass direction that the gerbils could use to guide directional responding. In the second experiment, we used a different task, in which two landmarks were required in order to locate the target. In this procedure, the relationship between the two landmarks was consistent from trial to trial, and all other sources of direction, although internally consistent, were disengaged from the landmark locations. In addition, and unlike any previous experiment of this kind, the landmark array was rotated randomly from trial to trial with respect to directional information from other cues.

\section{Method}

Subjects. Ten male Mongolian gerbils were used in this experiment. The details of housing and feeding were exactly the same as those for Experiment 1.

Apparatus. The same testing box and landmarks were used as those in Experiment 1.

Procedure. The gerbils received habituation sessions with the apparatus as described in Experiment 1. During the training phase, the two landmarks were placed in a random location and at a random orientation within the box, but the distance between the two landmarks was always $10 \mathrm{~cm}$. The food target was placed at the third vertex of an equilateral triangle formed with the two landmarks (in other words, the food target was $10 \mathrm{~cm}$ from each of the two targets). For each gerbil, the target was always placed on the same side of the triangle. Hence, if the landmarks are considered as defining the base of the triangle, then, viewed from the target, one landmark would be consistently to the left and the other to the right. Half of the animals were trained with the gray landmark on the left and the 


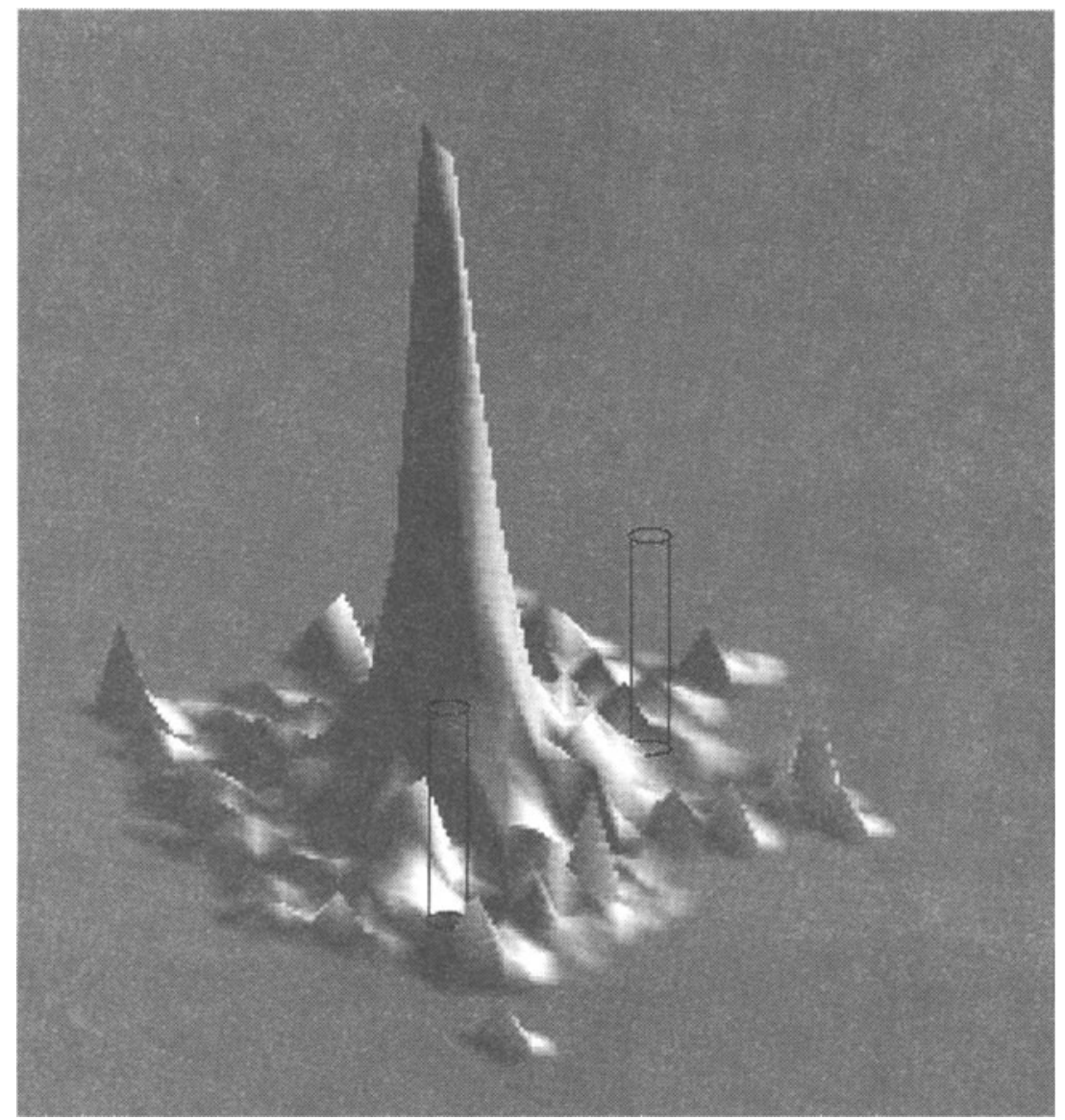

Figure 3. Contour plot illustrating the frequency distribution of all the time-sampled locations of all the animals on all the trials in Experiment 2. The positions of the landmarks are superimposed as wireframe drawings. As the two landmarks were freely rotated and translated from trial to trial, location information was normalized. The high peak in the figure lies directly over the target location and illustrates the overall precision of searching at the target location.

silver one on the right, and the other half were trained with the opposite arrangement. As in Experiment 1, training was continued until the gerbils were able to reliably retrieve the food in less than $10 \mathrm{sec}$ per trial on $75 \%$ of the trials. Test trials were carried out over a period of 3 days, with two probe trials per day. For each probe trial, the gerbils were placed into the apparatus at a random location and were videotaped for $30 \mathrm{sec}$. As in Experiment 1, no food was present on the probe trials. Just as on training trials, there was no consistent relationship between the location or orientation of the landmarks and the location of any other intra- or extramaze information or with any navigational signal derived from dead reckoning.

Analysis. Videotaped trajectories on the probe trials were converted to movie files, using a commercially available frame grabbing interface (LG-3, Scion Corporation), and $x y$-coordinates of the tip of the animals' snouts were recorded at $1 / 2-\mathrm{sec}$ intervals. This method, which had not been available for the analysis of the results from Experiment 1, made it possible for us to quantify the temporal properties of the target search, as well as its spatial properties.

\section{Results and Discussion}

The gerbils were able to learn to search at the correct target location in this experiment. In order to show the precision of the target search, we computed a surface plot of exploration time in the part of the field close to the landmark (Figure 3). This plot represents the summed performance of all 10 animals in this experiment, and the high peak is located directly over the correct target location. For statistical analysis, we used a method similar to the one described previously. As for a dependent measure, we used the total elapsed time (estimated by counting time samples) that a gerbil spent within $5 \mathrm{~cm}$ of the target location and the total time spent within $5 \mathrm{~cm}$ of the antitarget location (with respect to the two landmarks, the location that was mirror symmetric to the target location). A two-way repeated measures ANOVA revealed that the gerbils were significantly more likely to search at the target location than at the antitarget location $[F(1,8)=25.221$, $p<.001]$, but the trial and interaction effects were both nonsignificant.

There are a number of possible strategies that a gerbil could use to find the correct target location in this task. One possibility, most in keeping with the idea of a land- 


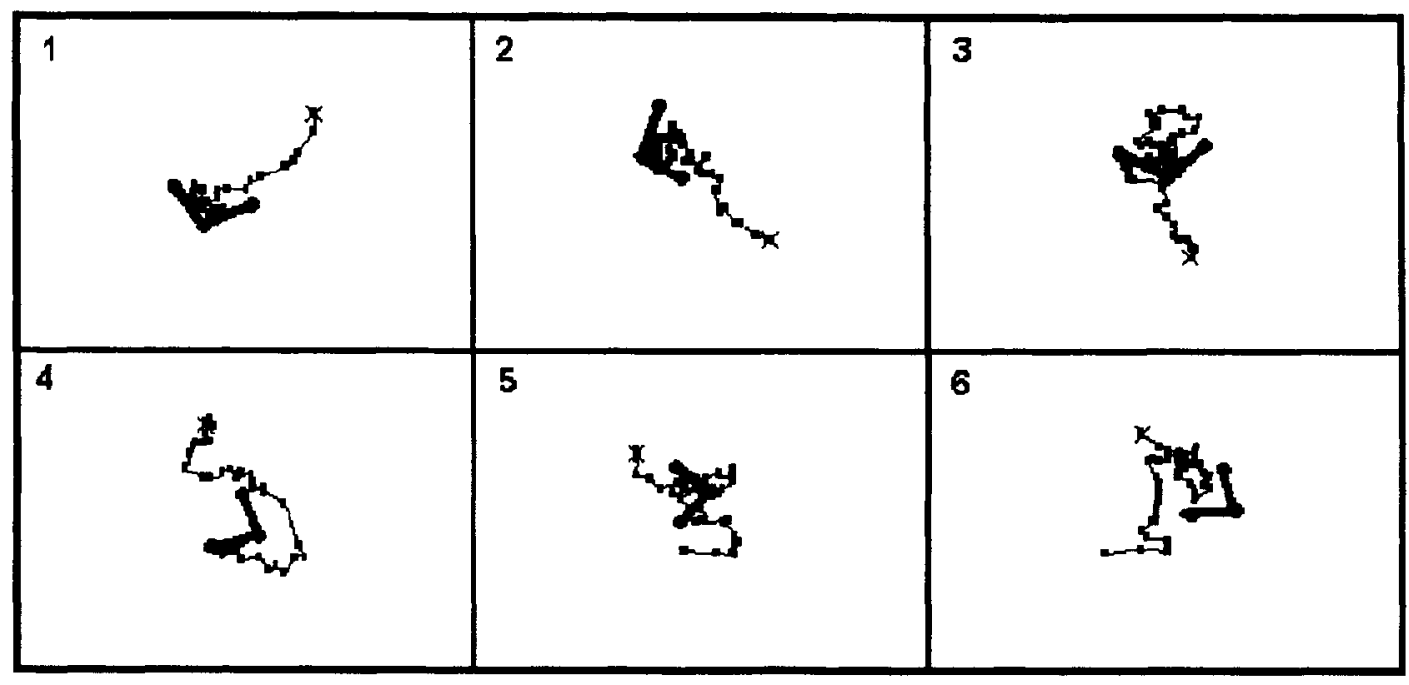

Figure 4. Schematic diagrams showing the performance of 1 gerbil on six different probe trials. Time-sampled location points are represented by small squares, with connecting lines illustrating the animal's trajectory. A small $X$ is used to illustrate the starting point of a trial. The landmark-target array is represented by a $V$, with the vertex of the $\mathrm{V}$ representing the target location. The illustration shows that there is little evidence suggesting that this gerbil adopted a standard trajectory of approach to walk to the target location.

mark as a feature of a map, would be for the gerbils to extract a positional vector from the locations of the landmarks and then to walk directly to the target location. Another possibility, however, would be for the gerbils to treat the two landmarks together as a single composite object and to then search for the "front" of the object, perhaps by walking around the apparatus to obtain a series of different local views. Indeed, during training, we sometimes believed that the gerbils might have been adopting such a strategy, because they appeared to walk around the perimeter of the apparatus before approaching the targets. Figure 4 shows a diagrammatic representation of the single-trial trajectories that were carried out by 1 representative gerbil. What is most obvious from these plots is that this gerbil did walk more or less directly to the immediate vicinity of the landmarks. Of particular note is the contrast between Trial 1 and Trial 3. In these two cases, the gerbil's starting point is on opposite sides of the triangle, yet the approach to the target location is quite direct in each case, with the gerbil walking between the landmarks, when necessary, to obtain the target.

These findings, similar to those presented by Biegler and Morris (1996a), suggest that gerbils are able to use an array of two landmarks that maintain a constant spatial relationship to each other and to a target in order to navigate directly and precisely to the target location. An examination of the trajectories of the animals to the target suggests that this feat of navigation is not achieved by attempting to match one particular local view of the targets with a stored representation, but it does not rule out the possibility that, as others have suggested (Leonard \& McNaughton, 1990), gerbils consult a library of different local views to help them plot a course to the target location.
These findings extend those of Biegler and Morris (1996a) by demonstrating that gerbils are able to solve the two-landmark problem even when the landmarks rotate randomly from trial to trial with respect to all other sources of location and direction information. There is some suggestion in the work of Biegler and Morris (1996a) that accuracy in determining distance and direction from landmarks may be differentially influenced by certain manipulations of the arrangement or the stability of the landmark arrays. In this context, the present experiment constitutes important first evidence that the position and orientation of an array of two landmarks can be used by gerbils to define target location without contributions from geometry or extramaze cues.

\section{EXPERIMENT 3}

There is now a substantial literature on the connections (Chandler, King, Corwin, \& Reep, 1992; Corwin \& Reep, 1998), physiology (Chen, Lin, Barnes, \& McNaughton, 1994; Chen, Lin, Green, Barnes, \& McNaughton, 1994; McNaughton et al., 1994), and function (Kesner et al., 1987; Kolb, 1990; Kolb et al., 1994; Kolb \& Walkey, 1987; Long, Mellem, \& Kesner, 1998; McDaniel, Compton, \& Smith, 1994; McDaniel, Williams, Attaway, \& Compton, 1998) of an area of the rodent cortex thought by many to be homologous with the posterior parietal cortex in primates. Although there is widespread agreement that this area of the rodent cortex makes a contribution to spatial behavior, there is almost no agreement on the exact nature of this contribution. Earlier accounts of spatial deficits after parietal lesions have been based on deficits in the Morris water-maze task (Kolb \& Walkey, 1987), in the 


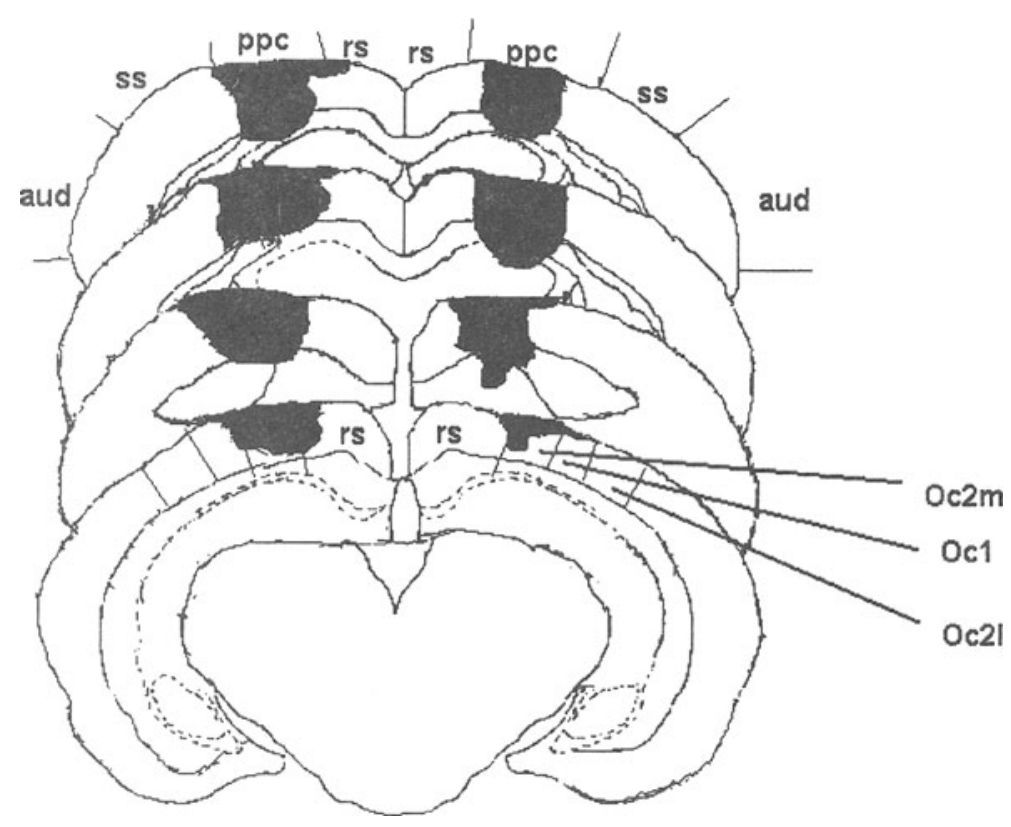

Figure 5. A schematic reconstruction of a typical lesion from Experiment 3. Cytoarchitectonic boundaries are drawn by using data from Shook (1983) and Cudmore, Long, and Ellard (1993). Abbreviations: ppc, posterior parietal cortex (rostral junction of Areas $18 \mathrm{a}$ and $18 \mathrm{~b}$, according to Caviness (1975) and Shook; rs, retrosplenial cortex (Areas 29c and 29d); Ocl, primary visual cortex (Area 17); Oc2l, lateral extrastriate cortex (Area 18a); $0 \mathrm{c} 2 \mathrm{~m}$, medial extrastriate cortex (Area 18b); ss, somatosensory cortex (Krieg's Areas 1 and 3); aud, auditory cortex (Areas 22 and 41).

cheeseboard task (DiMattia \& Kesner, 1988b), in some versions of the radial-arm maze task (DiMattia \& Kesner, 1988a), and on various tests involving measures of locomotion in open fields (Poucet \& Benhamou, 1997). Although most studies have focused on the acquisition of spatial habits, those few studies in which retention has been studied have also found deficits. Indeed, Poucet (1993) has argued that the parietal cortex is likely to be the locus of metric spatial maps of navigated space, suggesting that one might expect to find deficits in retention.

Given this background, it seemed reasonable to test the effects of parietal lesions on performance of the twolandmark tasks described above. Because each of these two tasks requires a different subset of information (landmark identification + compass direction, in one case, and compass direction derived from landmark identification, in the other), the pattern of deficits observed after parietal lesions might help to shed light on the contribution of the parietal cortex to these aspects of spatial behavior.

\section{Method}

Subjects. The subjects were 8 Mongolian gerbils ranging in age from 6 to 12 months at the time of testing. The gerbils were housed individually in polypropylene cages and were kept on a 12:12-h light:dark schedule. The animals were food restricted during landmark training but were given food at least once every $24 \mathrm{~h}$ and were maintained at $85 \%$, at least, of their free-feeding weight.
Training. Landmark training was conducted in the same apparatus as that described in Experiments 1 and 2, and exactly the same procedure as that in Experiment 1 was used. The gerbils were trained to respond to one of two cylinders, and the food was always located $10 \mathrm{~cm}$ "north" of one of the landmarks on training trials. On test trials, probe trials were randomly inserted into a series of six regular trials. On probe trials, no food was present in the test field. The gerbils were videotaped from above. The criterion for training was that the gerbils should be able to consistently find the food reward within $10 \mathrm{sec}$ of introduction into the test field on $75 \%$ of the trials.

Surgery. Following training to criterion, the gerbils were treated prophylactically with Septra antibiotic in their drinking water for $48 \mathrm{~h}$. Following this, they were anesthetized with sodium pentobarbital $(60 \mathrm{mg} / \mathrm{kg})$ and treated with atropine sulfate $(10 \mathrm{mg} / \mathrm{kg})$ and buprenorphine (Temgesic, $0.3 \mathrm{mg} / \mathrm{kg}$ ). Their eyes were moistened with saline gel (Dura Tears), and the scalp overlying the area of the incision was shaved. The animal was placed into a stereotaxic instrument, and the scalp was pretreated with a surgical scrub and a disinfecting solution. A $1-\mathrm{cm}$ midsaggital incision was made, and a craniotomy overlying the posterior parietal cortex was made with a dental drill. Posterior parietal cortical lesions were made by suction aspiration, removing all tissue rostral to the primary visual cortex and caudal to the somatosensory cortex, according to the cytoarchitectonic map of the gerbil cortex of Shook (1983) and our own observations (Cudmore, Long, \& Ellard, 1993). Following aspiration, the cavity was filled with gelfoam to promote hemostasis, and the incision was sutured closed. The animal was maintained on moist heat until it could right itself and then was returned to the home cage for a recovery period of 5-7 days. 


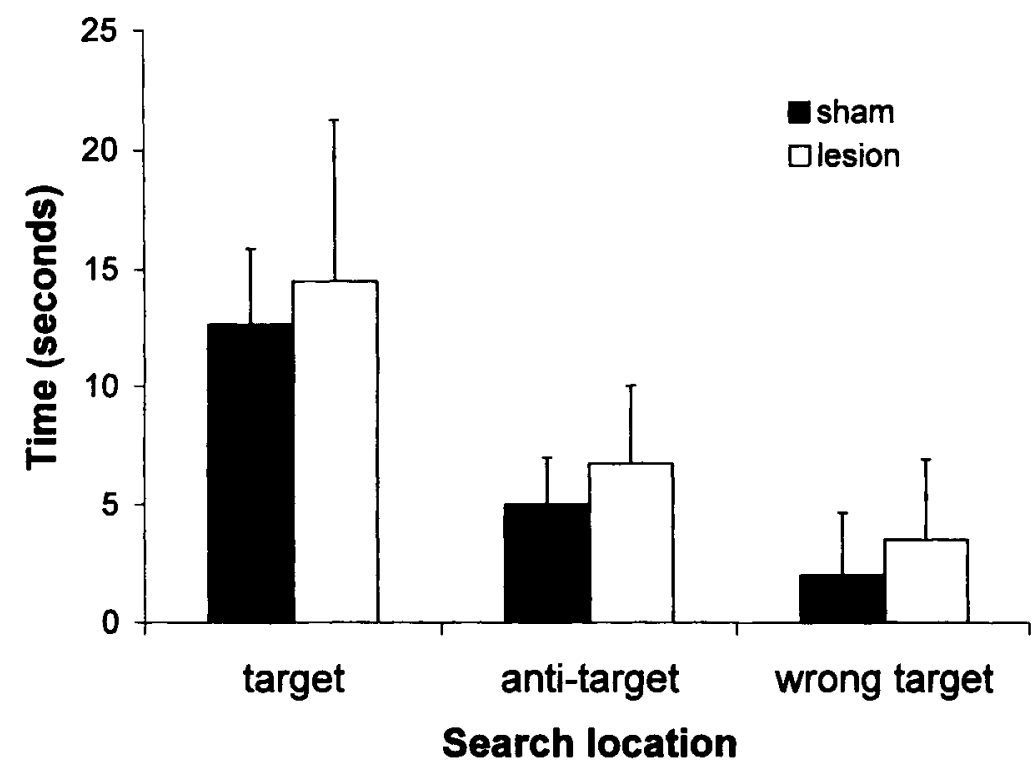

Figure 6. A histogram showing the average time spent within $5 \mathrm{~cm}$ of the target in Experiment 3, as compared with time spent within $5 \mathrm{~cm}$ of the antitarget location ( $5 \mathrm{~cm}$ south of the positive landmark) and the wrong target location $(5 \mathrm{~cm}$ north of the negative landmark). Error bars represent standard errors of the mean.

For the sham group, all the procedures and drug treatments that were used with the lesion animals were repeated, but no craniotomy was made, and no lesions were performed.

Postoperative testing. Postoperative testing was identical with that described for Experiment 1. Each animal received test trials on 3 consecutive days. On each day, the animal received eight trials, two of which were designated as probe trials. On probe trials, the animals were placed in the box with the landmarks but with no food reward, for a period of $30 \mathrm{sec}$. Their movements were videotaped and quantified, using the methods described for Experiment 1 .

At the conclusion of testing, the gerbils were sacrificed with an overdose of sodium pentobarbital (Euthanyl, $240 \mathrm{mg} / \mathrm{kg}$ ) and perfused transcardially with physiological saline followed by a $4 \%$ formalin solution. The brains were extracted and left in a solution of $4 \%$ formalin for at least $48 \mathrm{~h}$, following which they were frozen and sectioned at 40 microns, using a sliding microtome. Every third section was mounted on gelatin-coated slides, and three-dimensional reconstructions of lesion sites were prepared, using a videocamera and a Scion LG-3 image capture board.

\section{Results and Discussion}

Anatomical results. Figure 5 shows a schematic reconstruction of the lesions that were carried out in this experiment. According to the map of the gerbil neocortex given by Shook (1983), our lesions included either all or almost all of the cortical tissue rostral to the primary visual cortex (Area 17) and caudal to the somatosensory cortex (Areas 2 and 3 ). In confirmation of this, we were able to find the rostral border of Area 17 by looking for the distinctive band of cells in Layer IV, which is usually easily visible in gerbils. Inspection of the thalamus revealed the presence of degeneration in the nucleus lateralis posterior and also in the dorsal lateral geniculate nu- cleus. Because such degeneration is very sparse and difficult to identify, the more reliable indicators of the locus of damage in these animals were those based on cortical cytoarchitecture. All the histological evidence was consistent with damage to the area of the cortex lying between the primary visual and the somatosensory cortex, which is usually the area that is identified as being analogous to the posterior parietal cortex (DiMattia \& Kesner, 1988a, 1988b; Kolb \& Walkey, 1987).

Behavioral results. The search patterns of the gerbils were quantified in the manner described for Experiment 1 , and the total time spent searching within $5 \mathrm{~cm}$ of the target location, the antitarget location, and the wrong target location were quantified and are shown for both groups in Figure 6 . The main finding was the complete absence of a deficit in the parietal group. There was no evidence of a treatment effect $[F(1,5)=0.49, p>.1]$ or of an interaction effect $[F(2,10)=0.005, p>.1]$, and search patterns were tightly confined to the target location in both groups $[F(2,10)=22.3, p<.001]$.

These findings suggest that the ability of gerbils to find a location in space by using a combination of an unstable landmark and a compass direction derived from extramaze information is independent of the cortical area that has been defined as the rodent analogue of the posterior parietal cortex.

Given that rodents with parietal lesions have been reported to have some degree of deficit in virtually every spatial navigation task in which they have been tested, the absence of a deficit in the present task is striking. There are a number of possible explanations for the ab- 
sence of deficits in this task, but we will postpone discussion of most of these possibilities until after presentation of the results of the fourth experiment.

\section{EXPERIMENT 4}

Findings from the previous experiment suggest that gerbils with lesions of the parietal cortex do not have difficulty localizing a target that is always placed at a constant distance and in a constant direction from an unstable landmark. In this experiment, we set out to use the same procedure as the one described for Experiment 2 to examine the effects of parietal lesions on a different variant of the landmark task. In this variant, it will be recalled, solution of the task requires the gerbils to find a location that is defined purely on the basis of the location of two landmarks in the testing box. Although other sources of location information are present in the setup and are internally consistent, they cannot be used to find the target, since the landmark array moves randomly from trial to trial.

\section{Method}

Subjects. Eight Mongolian gerbils were used as subjects in this experiment. All the gerbils were between 3 and 6 months of age at the time of testing.

Procedure. Training and testing protocols were exactly the same as those described for Experiment 2. Gerbils were trained to find food that was always located at the third vertex of an equilateral triangle formed by two distinct landmarks. Once the gerbils could complete this task to criterion (retrieval of food within $10 \mathrm{sec}$ on $75 \%$ of the training trials), they received either a sham procedure or a lesion of the posterior parietal cortex, using the same procedure as that described for Experiment 3. Following 5-7 days of recovery, the gerbils were tested over 3 consecutive days, receiving two probe trials on each test day. Locations of the landmarks and the starting positions of the animals were randomized from trial to trial.

At the conclusion of testing, the gerbils were sacrificed with an overdose of sodium pentobarbital (Euthanyl, $240 \mathrm{mg} / \mathrm{kg}$ ) and were perfused transcardially with physiological saline followed by a $4 \%$ formalin solution. The same histological procedures were followed as those described for Experiment 3.

\section{Results and Discussion}

Anatomical results. The lesions were similar to those reported in the previous experiment. The damaged areas of the cortex included all of the cortex rostral to Area 17, extending rostrally to the somatosensory cortex and, in two cases, including some parts of the somatosensory cortex. Degeneration was observed in the nucleus lateralis posterior in all animals.

Behavioral results. The performance of the animals in this task was quantified as described for Experiment 2 by counting the number of time samples in which the gerbils were within $5 \mathrm{~cm}$ of the target location on the probe trials and by comparing these numbers with times spent at an antitarget location on the wrong side of the landmark array. Overall, the results were very similar to those reported for normal animals in Experiment 2. Statistical analysis with a repeated measures ANOVA showed a sig- nificant effect of target location, so that gerbils spent significantly longer in proximity to the target than to the antitarget position $[F(1,6)=7.47, p<.05]$. The treatment effect and all interaction effects were nonsignificant. Just as in Experiment 3, the main finding in this experiment is the absence of a parietal deficit in a landmark task that, unlike the previous task, relies entirely on the ability of animals to extract a compass direction from the orientation of two freely rotated and translated landmarks. Again, considering the quite consistent finding of deficits in spatial navigation tasks after parietal lesions, this outcome is counterintuitive.

Most studies that show deficits in navigational performance following lesions of the parietal cortex have employed latency measures (e.g., Kolb \& Walkey, 1987). In order to use a comparable test, we compared the times at which each animal was first within $5 \mathrm{~cm}$ of the target or the antitarget location in each of the two lesion groups. There were no significant differences between shams and parietals with respect to treatment, trial, or any interaction of these factors. Indeed, parietal animals were slightly faster at finding the target than were the shams.

In this experiment, as in the previous one, we found no evidence for any deficit in a task that is not only spatial in a general sense but that could be characterized as an allocentric spatial task. It is difficult to imagine that there is any way to solve the task given in Experiments 2 and 4 , other than to rely on landmark information to provide both a positional cue and a directional vector. Since the results of many other studies have suggested that tasks that require the use of such information are integrally dependent on the parietal cortex, our task is to account for our discrepant findings.

One possibility is that the dependent measure that was used in this study was not sufficiently sensitive to reveal a deficit. The experiments most directly comparable with the one reported here are those based on the landmark variant of the water-maze task. In such experiments (Crowne, Novotny, Maier, \& Vitols, 1992; Kolb et al., 1994; Kolb \& Walkey, 1987), the dependent measures that are usually employed are initial heading and latency. Generally, rats with parietal lesions show deficits with both of these measures. Because of the method of quantifying video data that was employed in some of these experiments, it was not easily possible to extract either latency or heading information on probe trials, but even if such measures were to show differences between groups, it would still be difficult to dispute the clear finding illustrated in Figure 6, that gerbils with parietal lesions showed searches that were concentrated at the correct target location and, so, could not have had major difficulties in locating the target. In addition, latency to find the target was examined in the fourth experiment, and there was no evidence of a deficit in parietal animals.

A second possibility is that the gerbils in this task did not show a deficit because we tested retention of a spatial habit, rather than acquisition, as is more commonly assayed. However, there is no a priori reason to suppose that 
this particular aspect of our test protocol would have made this much of a difference. When retention has been tested (DiMattia \& Kesner, 1988b), clear deficits have usually been reported.

A third possibility is that our lesions of the parietal cortex were either too small or incorrectly placed. Indeed, Poucet and Benhamou (1997) have argued that much of the variable outcome of the studies of the rodent parietal cortex can be accounted for on the basis of variability in lesion size and location. This problem is even more worrisome in our experiment, because most of the extant anatomical literature on this part of the rodent cortex is based on studies of the neuroanatomy of the rat.

There are several reasons to believe, however, that our lesions were analogous to those that have been carried out in other rodents and have been defined as lesions of the posterior parietal cortex. This assessment was based on several considerations. First, the area that was targeted for the lesions was part of the rostral arm of an area defined by Shook (1983) as Area 18a. Following similar work in the mouse, carried out by Caviness (1975), Shook eliminated Krieg's (1947) Area 7. However, the consensus of modern opinion (see Corwin \& Reep, 1998, for review) suggests that the area rostral to the primary visual cortex and caudal to the primary somatosensory cortex is better considered as a polysensory area similar in connections and organization to the posterior parietal cortex in primates. Second, a detailed inspection of the cytoarchitecture of the cortex at areas adjacent to the lesion suggests that we were successful in removing much of the posterior cortex that was rostral to the primary visual cortex, although our lesions did not appear to extend laterally as far as those employed in some other, similar studies using rats (e.g., Kesner et al., 1987; Kesner et al., 1989). Since the visual cortex is fairly easy to recognize in gerbils (Cudmore et al., 1993), we are confident that our lesions fell just rostral to the primary visual cortex. Third, some previous work in our laboratory has yielded results that are in accord with the predicted effects of parietal lesions. Ellard and Dias (1994) reported a parietal deficit in a spatial location dishabituation task similar to a task designed by Thinus-Blanc and Ingle (1985), and Ellard and Sharma (1996) reported a novel deficit in spatial context discrimination in a visuomotor task following parietal lesions. Collectively, these considerations suggest to us that our lesions are comparable with those that have been described as posterior parietal lesions in rats.

A fourth possible reason for the lack of deficits after parietal lesions in these experiments is that the task requirements differ in some way from those that have previously been shown to produce deficits in parietal animals. In particular, landmark variants of the Morris task usually require an animal to find a target that is fairly distal to the landmark and whose exact location serves as a rough guide to the location of the target. In most cases, control rats in the Morris landmark task swim directly to- ward the platform, whereas parietal rats appear to adopt a nonspatial strategy that involves swimming around the perimeter of the pool until they reach the target (Kolb \& Walkey, 1987). In our task, because the target was not located at a consistent distance or bearing from any other object in the test setup other than the landmark itself, gerbils could only find the target by measuring a distance (albeit a small one) from the landmark. This seems to have been a task that parietal animals had little or no difficulty carrying out. Although they used a go/no-go procedure rather than a navigational task, the present finding is reminiscent of the evidence for preserved computation of both egocentric and allocentric distance reported by Long and Kesner $(1996,1998)$.

In order to explore whether the nature of the landmark task itself-and, particularly, the relationship between the landmarks and the target - was responsible for the failure to find a deficit after parietal lesions, we carried out another experiment, in which the parameters of the task were altered to more closely resemble some tasks that show deficits after parietal lesions.

\section{EXPERIMENT 5}

In the Morris water-maze task, a rat is trained to find a submerged platform whose location does not usually vary from trial to trial. Although this is not often tested explicitly, the assumption is that the rat learns to navigate to the platform by building a spatial representation of the platform's location with one or more of the objects that are arrayed throughout the testing room. Similarly, in radial maze experiments, the assumption is usually made that the animal can distinguish between arms by recognizing the spatial arrangement of objects in the distal environment. Systematic tests of this have shown that rats find locations on the basis of the relative locations of arrays of targets (Suzuki et al., 1980) and that, when presented with both intra- and extramaze landmarks, they usually regulate search behavior in closer accord with extramaze landmarks than with intramaze landmarks (Kraemer, Gilber, \& Innis, 1983). Indeed, in some circumstances, rats appear to show a striking inability to take advantage of prominent intramaze information, rather than somewhat more subtle extramaze information or test field geometry (Cain, Beiko, \& Boon, 1997; Cheng, 1986). In accord with this, evidence from hippocampal place cells (O’Keefe \& Conway, 1980) suggests that place fields are built on the basis of configurations of arrays of distal landmarks but that, to some extent, such fields can survive manipulation or removal of subsets of distal information, so long as certain minimal amounts of information remain (Etienne, Joris-Lamber, DahnHurni, \& Reverdin, 1995; Worden, 1992). This finding makes good adaptive sense, since one would expect animals to be able to maintain heading vectors even in the face of changing and uncertain circumstances. In general, it has been argued that more salience is given to large, 
distant landmarks than to closer, smaller landmarks, but this can depend on the context in which the landmarks are presented (Barry \& Francq, 1982; Bennett, 1993; Etienne et al., 1995; Etienne, Joris, Maurer, \& Teroni, 1990). This, too, makes good sense for an animal, in that the larger distant objects are much more likely to form a part of the permanent topography of the animal's environment (such as a tree or a hill), and small, close objects (stones, small plants) are more likely to be unstable. If, as has been argued by some (e.g., Poucet, 1993), the parietal cortex is a major part of the brain circuitry that represents spatial information in the form of a map, one might expect to see such maps being based on distal environmental objects, rather than on close objects. If this is true, we would expect a task in which target locations are not defined on the basis of unstable proximal landmarks to be more prone to disturbance by parietal lesions. We designed such a task by removing the landmarks from the test field and training the animals to find food at one particular location in the field, which was defined jointly by the geometry of the field and the locations of an array of distal landmarks.

\section{Method}

Subjects. Nine Mongolian gerbils were the subjects in this experiment. All the gerbils were housed and maintained in the same manner as that described for previous experiments.

Procedure. On the first day of training, the gerbils were introduced into the test field for a period of $30 \mathrm{~min}$, and they were allowed to explore and forage for food. Following this day of habituation, each day of training consisted of a series of discrete trials. On each trial, a small morsel of lab chow was placed in the field at a location $30 \mathrm{~cm}$ from one edge and $30 \mathrm{~cm}$ from the adjacent edge, using a cardboard template to ensure accurate positioning. The animal was placed into the box and left for a period of $1 \mathrm{~min}$ or until it found the food. Initially, a small area of bedding was cleared from the vicinity of the target, in order to make the task easy to learn. Once the animals had begun to learn the task, the food morsel was buried under bedding. Prior to each trial, the bedding in the apparatus was churned randomly, in order to minimize olfactory traces that might build up near the target location. Two target locations were chosen, and the animals were randomly assigned to one of the two targets. With respect to the orientation of the test box and the walls of the testing room, the two targets were rotated $180^{\circ}$ from one another. A set of prominent features was attached to the walls of the testing room, including large geometric shapes and a black curtain covering almost all of one wall. In addition, during training trials, the experimenter sat on a stool that was always located in the same place in the room. From most locations in the testing field, the top of the experimenter's head would have been visible to the gerbils.

Surgical procedure. Once the gerbils reached training criterion, defined as successfully finding food targets on $70 \%$ of the trials in less than $10 \mathrm{sec}$ from release, they were divided randomly into two groups. One group received the sham procedure described above, and the other group received lesions of the parietal cortex.

Testing procedure. Following at least 5 days of recovery, the gerbils were retested for retention of landmark information. Test sessions consisted of 4 consecutive days of testing. On each day, the gerbils were presented with eight trials, two of which were probe trials in which there was no food reward in the apparatus. On each of the eight trials, the gerbils were placed either in one of the four corners of the apparatus or at the midpoint of one of the four walls. Over the 4 days of testing, the gerbils received one probe trial from each of the eight starting positions. The trials were videotaped, and quantitative positional analysis was conducted on probe trials, using methods similar to those described for previous experiments.

\section{Results and Discussion}

Anatomical results. Parietal lesions were similar to those described for previous experiments.

Behavioral results. The search patterns on probe trials were analyzed, using methods similar to those described for the previous experiments. The total amount of time spent within $5 \mathrm{~cm}$ of the target location was measured by reconstructing the location of the head on videotape sampled at 500-msec intervals. For comparison purposes, the amount of time spent near the target location was compared with the amount of time spent at an antitarget location, which was defined as a location at the same distance from two of the walls of the apparatus but rotated by $180^{\circ}$ (in other words, the antitarget of Target A was Target Location B, and vice versa). Figure 7 shows search times at the target and the antitarget locations for the control and the parietal animals. A statistical analysis showed a significant interaction between treatment and target $[F(1,7)=14.578, p<.01]$. Further analysis showed that the control animals spent significantly more time in the vicinity of the target than of the antitarget $[F(1,3)=81.00$, $p<.01]$ but that the parietal animals did not $[F(1,4)=$ $3.347, p>.1]$. We also measured the latency to reach the target in probe trials, since this is a measure that is more directly comparable with other reported deficits in navigation performance after parietal lesions in the Morris water-maze task (Crowne et al., 1992; Kolb et al., 1994; Kolb \& Walkey, 1987). Although parietal animals took, on average, somewhat longer to reach the target than did controls $(20.26$ vs. $14.15 \mathrm{sec})$, these differences fell short of statistical significance. This is not surprising, however, since probe trials were strictly limited to $30 \mathrm{sec}$ and many lesion animals did not find the target at all during this time interval. These results provide the clearest evidence in this experimental series for deficits in spatial navigation after lesions of the parietal cortex, and they confirm our hypothesis that parietal deficits are exacerbated in tasks in which the relationship between a target location and the landmarks that define that location are distal to the target and/or in tasks in which the target location is defined by a complex array of distal landmarks.

A possible counterargument to this suggestion is that the parietal gerbils showed deficits in the final experiment because this task was more difficult for them than were the tasks involving navigation with the use of intramaze landmarks. The appearance of a deficit would then be accounted for in accord with the suggestion of Save, Poucet, Foreman, and Thinus-Blanc (1998) that parietal animals show deficits in tasks that require effortful processing of spatial information. Such an alternative explanation does not fit well with the finding that, when both kinds of landmarks are available, extramaze landmarks are used preferentially (Suzuki et al., 1980). In addition, the earlier tasks required animals to disembed unstable land- 


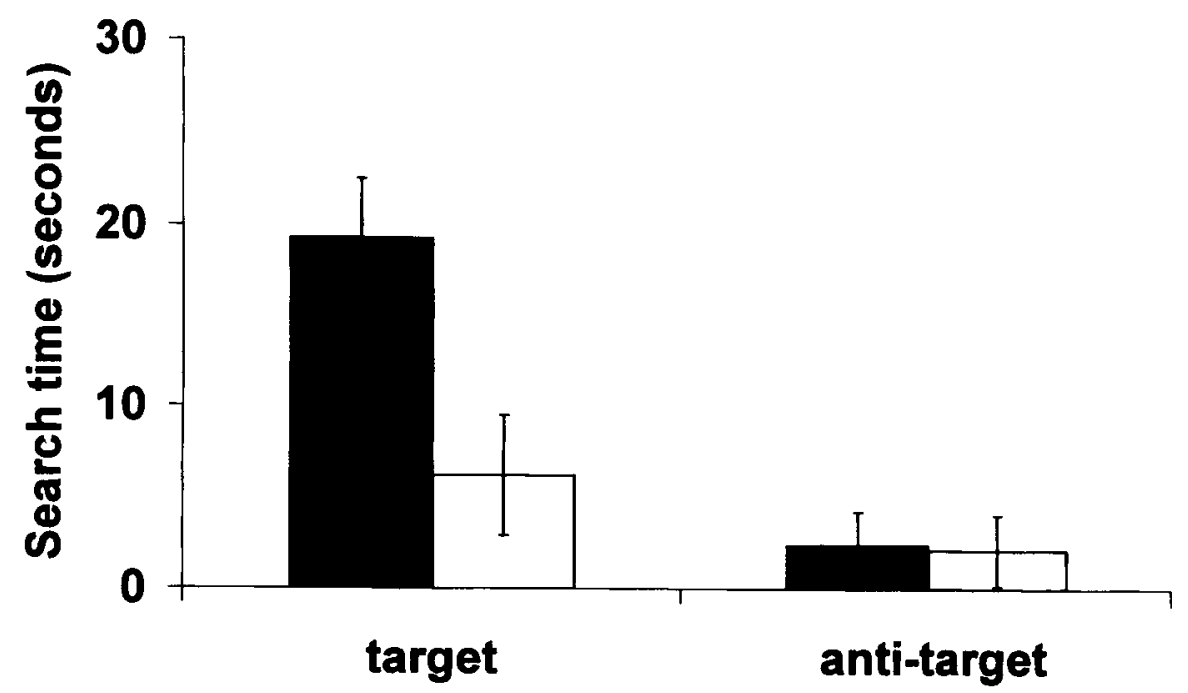

\section{Location}

Figure 7. A histogram showing the average amounts of time spent at the target or the antitarget (location in the test field at a location rotated $180^{\circ}$ from the target location but at the same distance from the walls of the apparatus). Filled bars represent the performance of the sham animals, and open bars show the performance of the animals with parietal lesions. Error bars represent standard errors of the mean.

mark-target relationships from a context of stable spatial cues. This would seem to be a somewhat more difficult task than simply finding a location defined by a set of stable extramaze landmarks.

\section{GENERAL DISCUSSION}

Collectively, the results from the series of experiments that we report in this paper make two original contributions. The first of these concerns the extension of the findings of Biegler and Morris (1993, 1996a, 1996b) concerning the conditions under which rodents are able to navigate to targets by using unstable landmarks. As was described earlier, Biegler and Morris have argued that landmarks that occupy locations that are unpredictable with respect to location information derived from other sources of information exert diminished control over search behavior and, under certain conditions, are completely ineffective navigational cues, even when their location reliably predicts the location of a reward. One of the main reasons for Biegler and Morris' preoccupation with this issue is a concern with determining whether spatial learning follows rules that are qualitatively different from general learning principles (Gallistel, 1990; O'Keefe \& Nadel, 1978) or not (Dickinson, 1980; Mackintosh, 1983). In this context, Biegler and Morris' demonstration that instability weakens the associative strength between a landmark and a target location is counter to some of the central tenets of modern learning theory (Rescorla, 1968; Wagner, Logan, Haberlandt, \& Price, 1968). Because we did not compare stable with unstable landmarks, our ex- periments have no impact at all on this argument, but our finding that gerbils can use unstable landmarks to find targets in two different conditions confirms Biegler and Morris' suspicion in their 1996 papers that it was likely that the failure of rats in the 1993 experiments to find targets with unstable landmarks was due to the vestibular disorientation procedure that they used. When all ambient sources of directional information within the testing room are kept coherent, gerbils are easily able to navigate to a target by using an unstable landmark. In addition, our findings extend those of Biegler and Morris, in that they demonstrate that gerbils can navigate to a target that is rotationally unstable, as well as translationally unstable. As was described previously, unless the landmark-target array both rotates and translates freely from trial to trial, it is impossible to claim that animals are navigating purely on the basis of landmark locations without using other sources of information (the geometry of the test field and/or the experimental room) to compute target locations. Our demonstration that gerbils search accurately with rotated landmark arrays constitutes the first such demonstration that we are aware of.

In a historical context that suggests that parietal lesions produce deficits in both the acquisition and the retention of virtually every spatial task that has been tested, our finding that such lesions do not seem to affect tasks that involve navigating to locations that are defined by either single landmarks or pairs of proximal landmarks is somewhat counterintuitive. Our suggestion here is that only those tasks that require animals to navigate to a place by using the locations of distal landmarks are likely to be 
affected by parietal lesions. The experiments that approximate most closely the landmark-learning task that we have used in the present work are landmark variants of the Morris water-maze task. As has been mentioned, such variants usually use markings on the walls of the maze as landmarks. These landmarks, although proximal in the sense that they are on the inside of the maze, are not really navigable three-dimensional objects in the same sense as the landmarks used in our studies. In addition, some investigators, in partial accord with Cheng's (1986) findings, have shown that such wall markings can be peculiarly ineffective in influencing navigational behavior (Cain et al., 1997). It could be that such cues have a somewhat ambiguous status, because they are neither clearly proximal (in the sense of being in navigable space) nor clearly distal.

Some recent accounts of the locus of the parietal deficit in rodents have focused on the possibility that cells in this area may be processing the relationship between movement-generated and visual information (ThinusBlanc, 1996). In particular, the neurophysiological evidence is suggestive of such a relationship, since many cells have firing correlates that involve particular kinds of head movements carried out in specific spatial contexts (Chen, Lin, Barnes, \& McNaughton, 1994; Chen, Lin, Green, et al., 1994; McNaughton et al., 1994). In addition, a recent study by Save and Moghaddam (1996) has shown that parietal rats show deficits in a task requiring them to navigate from a fixed location to a target in a darkened water maze. This constitutes evidence for a parietal contribution to egocentric mapping based on feedback from self-motion. Also in accord with such a notion is Kolb and Walkey's (1987) argument that parietal rats show deficits in a landmark version of the water-maze task because of difficulty extracting relevant (location of the landmark, which cued the location of the escape platform) from irrelevant (locations of stable distal landmarks) information, especially when the task involves learning a spatial relationship between the target and the landmark. It should be noted, however, that the deficit shown by rats in a visible landmark condition of the water-maze task was qualitatively different from that seen in the conventional task (this point is discussed by Thinus-Blanc, 1996, pp. 141-143). Parietal rats in the conventional water-maze task swam to the correct approximate location, whereas those in a visible landmark condition swam in circles in the pool at the correct approximate distance between the pool's edge and the target. This finding, replicated by Kolb et al. (1994), led them to claim that the posterior parietal cortex serves in "active guidance of the body through visual (and perhaps tactile space)" (p. 677), a claim that is not notably at odds with that made by Thinus-Blanc (1996).

Although our findings do not contradict any of this earlier work, they do suggest that the presence or, perhaps, the severity of the parietal deficit in spatial landmark navigation may depend on the nature of the relationship between the target location and the landmarks. Animals that can walk around the landmarks and navigate directly from them to the target location appear to be unaffected by lesions of the parietal cortex, but animals that need to use the appearances of sets of distal objects in order to find the target place show deficits after lesions.

These experiments leave many unanswered questions, which will need to be addressed in future work. Although it seems clear that distal landmark use is preferentially affected by parietal lesions, there are a number of important ways in which distal and proximal landmarks differ. The latter are navigable, in the sense described here, but they are also closer and, possibly, more distinct than distal landmarks, and they also typically occupy a lower position in an animal's visual field. It remains to future work to discover which of these differences might account for the present findings. In addition, the present experiments explored the effect of parietal lesions only on retention of landmark navigation learning and not on acquisition. Although both types of deficits have been reported in previous experiments, generally, acquisition deficits appear to be somewhat more severe than retention deficits. It will be important to confirm whether or not this pattern of findings is preserved in our landmark procedures.

\section{REFERENCES}

BARRY, R. E., \& FrancQ, E. N. (1982). Illumination preference and visual orientation of wild-reared mice. Animal Behaviour, 30, 339-344.

BenNetT, A. T. D. (1993). Remembering landmarks. Nature, 364, 293 294.

Biegler, R., \& Morris, R. G. M. (1993). Landmark stability is a prerequisite for spatial but not discrimination learning. Nature, 361 , $631-633$.

Biegler, R., \& Morris, R. G. M. (1996a). Landmark stability: Further studies pointing to a role in spatial learning. Quarterly Journal of Experimental Psychology, 49B, 307-343.

Biegler, R., \& Morris, R. G. M. (1996b). Landmark stability: Studies exploring whether the perceived stability of the environment influences spatial representation. Journal of Experimental Biology, 199, 187-193.

CAIN, D. P., Beiko, J., \& Boon, F. (1997). Navigation in the water maze The role of proximal and distal visual cues, path integration, and magnetic field information. Psychobiology, 25, 286-293.

Caviness, V. S., JR. (1975). Architectonic map of neocortex of the normal mouse. Journal of Comparative Neurology, 164, 247-264.

Chandler, H. C., King, V., Corwin, J. V., \& ReeP, R. L. (1992). Thalamocortical connections of rat posterior parietal cortex. Neuroscience Letters, 143, 237-242.

Chen, L. L., Lin, L.-H., Barnes, C. A., \& McNaughton, B. L. (1994). Head-direction cells in the rat posterior cortex: II. Contributions of visual and ideothetic information to the directional firing. Experimental Brain Research, 101, 24-34

Chen, L. L., Lin, L.-H., Green, E. J., Barnes, C. A., \& McNaughton, B. L. (1994). Head-direction cells in the rat posterior cortex: I. Anatomical distribution and behavioral modulation. Experimental Brain Re search, 101, 8-23.

Cheng, K. (1986). A purely geometric module in the rat's spatial representation. Cognition, 23, 149-177.

Collett, T. S., Cartwright, B. A., \& Smith, B. A. (1986). Landmark learning and visuo-spatial memories in gerbils. Journal of Compar ative Physiology $A, 158,835-851$.

CoRwIN, J. V., \& REEP, R. L. (1998). Rodent posterior parietal cortex as a component of a cortical network mediating directed spatial attention. Psychobiology, 26, 87-102.

Crowne, D. P., Novotny, M. F., Maier, S. E., \& Vitols, R. (1992). Effects of unilateral parietal lesions on spatial localization in the rat Behavioral Neuroscience, 106, 808-819.

Cudmore, L. J., Long, A., \& Ellard, C. G. (1993). Anatomical connec- 
tions of some visual cortical areas in the Mongolian gerbil (Meriones unguiculatus). Society for Neuroscience Abstracts, 19, 969.

Dickinson, A. (1980). Contemporary animal learning theory. Cambridge: Cambridge University Press.

DiMatTIA, B. V., \& Kesner, R. P. (1988a). Role of the posterior parietal association cortex in the processing of spatial event information. Behavioral Neuroscience, 102, 397-403.

Dimattia, B. V., \& Kesner, R. P. (1988b). Spatial cognitive maps: Differential role of parietal cortex and hippocampal formation. Behavioral Neuroscience, 102, 471-480.

Ellard, C. G., \& Dias, M. (1994). Two cortical visual systems in a rodent. Society for Neuroscience Abstracts, 20, 1742.

Ellard, C. G., \& Sharma, L. (1996). The effects of cortical lesions on object recognition in a visuomotor task in the gerbil. Behavioural Brain Research, 82, 13-22.

Etienne, A. S., Joris-Lamber, S., Dahn-Hurni, C., \& Reverdin, B. (1995). Optimizing visual landmarks: Two- and three-dimensional minimal landscapes. Animal Behaviour, 49, 165-179.

Etienne, A. S., Joris, S., Maurer, R., \& Teroni, E. (1990). Enhancing the impact of visual extra-maze cues in a spatial orientation task. Behavioural Brain Research, 38, 199-210.

Gallistel, C. R. (1990). The organization of learning. Cambridge, MA: MIT Press.

Kesner, R. P., DiMattia, B. V., \& CRUtcher, K. A. (1987). Evidence for neocortical involvement in reference memory. Behavioral \& Neural Biology, 47, 40-53.

Kesner, R. P., Farnworth, G., \& DiMattia, B. V. (1989). Double dissociation of egocentric and allocentric space following medial prefrontal and parietal cortex lesions in the rat. Behavioral Neuroscience, $103,956-961$.

KOLB, B. (1990). Posterior parietal and temporal association cortex. In B. Kolb \& R. C. Tees (Eds.), The cerebral cortex of the rat (pp. 459471). Cambridge, MA: MIT Press.

Kolb, B., Buhrmann, K., McDonald, R., \& Sutherland, R. J. (1994). Dissociation of the medial prefrontal, posterior parietal, and posterior temporal cortex for spatial navigation and recognition memory in the rat. Cerebral Cortex, 6, 664-680.

KOLB, B., \& WALKEY, J. (1987). Behavioural and anatomical studies of the posterior parietal cortex in the rat. Behavioural Brain Research, 23, 127-145.

Kraemer, P. J., Gilber, M. E., \& InNis, N. K. (1983). The influence of cue type and configuration upon radial-maze performance in the rat. Animal Learning \& Behavior, 11, 373-380.

KRIEG, W. J. S. (1947). Connections of the cerebral cortex: I. The albino rat: A topography of the cortical areas. Journal of Comparative Neurology, 84, 221-275.

Leonard, B., \& McNaughton, B. L. (1990). Spatial representation in the rat: Conceptual, behavioral and neurophysiological perspectives. In R. P. Kesner \& D. S. Olton (Eds.), Neurobiology of comparative cognition (pp. 363-422). Hillsdale, NJ: Erlbaum.

LONG, J. M., \& KESNER, R. P. (1996). The effects of dorsal versus ventral hippocampal, total hippocampal, and parietal cortex lesions on memory for allocentric distance in rats. Behavioral Neuroscience, $110,922-932$.

LONG, J. M., \& KESNER, R. P. (1998). Effects of hippocampal and parietal cortex lesions on memory for egocentric distance and spatial location information in rats. Behavioral Neuroscience, 112, 480-495.

LONG, J. M., MELleM, J. E., \& KESNER, R. P. (1998). The effects of parietal cortex lesions on an object/spatial location paired-associate task in rats. Psychobiology, 26, 128-133.

MaCKINTOSH, N. J. (1983). Conditioning and associative learning. Oxford: Oxford University Press.

Margules, J., \& Gallistel, C. R. (1988). Heading in the rat: Determination by environmental shape. Animal Learning \& Behavior, 16, 404-410.

MCDaniel, W. F., Compton, D. M., \& Smith, S. R. (1994). Spatial learning following posterior parietal or hippocampal lesions. NeuroReport, 5, 1713-1717.
McDaniel, W. F., Williams, L. B., Attaway, C. M., \& Compton, D. M. (1998). Turn-signal utilization by rats with either unilateral or bilateral posterior parietal cortex injuries. Psychobiology, 26, 143152.

McNaughton, B. L., Barnes, C. A., Gerrard, J. L., Gothard, K., Jung, M. W., KNIERIM, J. J., Kudrimoti, H., Qin, Y., SkagGs, W. E., Suster, M., \& Weaver, K. L. (1996). Deciphering the hippocampal polyglot: The hippocampus as a path integration system. Journal of Experimental Biology, 199, 173-185.

McNaughton, B. L., Mizumori, S. J. Y., Barnes, C. A., Leonard, B. J., Marquis, M., \& Green, E. J. (1994). Cortical representation of motion during unrestrained spatial navigation in the rat. Cerebral Cortex, 4, 27-39.

Mittelstaedt, M.-L., \& Mittelstaedt, H. (1980). Homing by path integration in a mammal. Naturwissenschaften, 67, 566-567.

O'KeEFE, J. (1976). Place units in the hippocampus of the freely-moving rat. Experimental Neurology, 51, 78-109.

O'KeEFE, J., \& ConWAY, D. H. (1980). On the trail of the hippocampal engram. Physiological Psychology, 8, 229-238.

O'KEEFE, J., \& NADEL, L. (1978). The hippocampus as a cognitive map. London: Oxford University Press.

Poucet, B. (1993). Spatial cognitive maps in animals: New hypotheses on their structure and neural mechanisms. Psychological Review, 100, 163-182.

Poucet, B., \& Benhamou, S. (1997). The neuropsychology of spatial cognition in the rat. Critical Reviews on Neurobiology, 11, 101-120.

RESCORLA, R. A. (1968). Probability of shock in the presence and absence of CS in fear condition. Journal of Comparative \& Physiological Psychology, 66, 1-5.

Save, E., \& Moghaddam, M. (1996). Effects of lesions of the associative parietal cortex on the acquisition and use of spatial memory in egocentric and allocentric navigation tasks in the rat. Behavioral Neuroscience, 110, 74-85.

Save, E., Poucet, B., Foreman, N., \& Buhot, M.-C. (1992). Object exploration and reactions to spatial and nonspatial changes in hooded rats following damage to parietal cortex or dorsal hippocampus. Behavioral Neuroscience, 106, 447-456.

Save, E., Poucet, B., Foreman, N., \& Thinus-Blanc, C. (1998). The contribution of the associative parietal cortex and hippocampus to spatial processing in rodents. Psychobiology, 26, 153-161.

Sноок, B. L. (1983). Functional organization of the gerbil's visual system: A move to revise traditional working hypotheses of brain organization. Unpublished doctoral dissertation, Brandeis University.

Suzuki, S., Augerinos, G., \& Black, A. H. (1980). Stimulus control of spatial behavior in the eight-arm maze in rats. Learning \& Motivation, 11, 1-18.

Thinus-Blanc, C. (1996). Animal spatial cognition: Behavioural and brain approach. Singapore: World Scientific.

Thinus-Blanc, C.. \& INGLE, D. J. (1985). Spatial behavior in gerbils (Meriones unguiculatus). Journal of Comparative Psychology, 99, 311-315.

Wagner, A. R., Logan, F. A., Haberlandt, K., \& Price, T. (1968). Stimulus selection in animal discrimination learning. Journal of $E x-$ perimental Psychology, 76, 171-180.

WORDEN, R. (1992). Navigation by fragment fitting: A theory of hippocampal function. Hippocampus, 2, 165-187.

\section{NOTE}

1. Note that this method of quantification, updated in most later experiments, does not allow us to reconstruct the animal's search path, but it does make it possible to examine the search pattern.

(Manuscript received June 16, 1999; revision accepted for publication September 15, 1999.) 